\title{
Autenticación de personas utilizando un clasificador SVM
}

\section{People authentication through a SVM classifier}

\author{
Aida A. Aparicio-Arroyo ${ }^{1}$ (D), Ivan Olmos-Pineda ${ }^{1}$ (D) , J. Arturo Olvera-López ${ }^{1}$ \\ ${ }^{1}$ Benemérita Universidad Autónoma de Puebla, Puebla, México. \\ aida.aparicio@alumno.buap.mx,iolmos@cs.buap.mx,aolvera@cs.buap.mx
}

(Recibido: 28 julio 2021; aceptado: 16 septiembre 2021; Publicado en Internet: 1 diciembre 2021)

\begin{abstract}
Resumen. En los últimos años, la autenticación de personas ha tomado un gran auge debido a los avances tecnológicos e investigaciones que se han desarrollado alrededor del tema. En este proceso se usan técnicas de visión por computadora que permiten procesar una imagen o video para determinar la identidad de una persona. En el presente artículo, se analizan trabajos relacionados con el proceso de autenticación de personas, haciendo un análisis profundo en los trabajos basados en Máquina de Vectores de Soporte (Support Vector Machines). De igual manera, se explican a grandes rasgos las diferentes etapas que conforman el proceso de autenticación de personas. Finalmente, se presenta un conjunto de experimentos realizados, utilizando una combinación de características basadas en color, textura y simetría, mientras que, para la etapa de clasificación se utiliza SVM. Esta combinación de características aunada con el clasificador, muestra ser una alternativa para la autenticación de personas.
\end{abstract}

Palabras clave: Extracción de características, SVM, Autenticación de personas.

Abstract. In recent years, people's authentication has taken a significant boom due to technological advances and research developed around the subject. In this process, computer vision techniques are used to process an image or video to determine a person's identity. In this article, we analyzed related works to the people authentication process, making a deep analysis in the works based on Support Vector Machines (SVM). In the same way, we roughly explained the stages that make up the process of people authentication. Finally, we present a set of experiments performed, using a feature combination based on color, texture, and symmetry. In contrast, SVM is used for the classification stage. This combination of features, together with the classifier, shows to be an alternative to people authentication.

Keywords: Feature extraction, SVM, People authentication.

Tipo de artículo: Artículo de investigación.

\section{Introducción}

Actualmente, el concepto de autenticación de personas se ha vuelto más relevante, debido al rápido avance de la tecnología y de sus diferentes aplicaciones. Existen diferentes formas de poder autenticar personas, a través de comandos de voz o leyendo la huella dactilar, pero en los últimos años, el usar características faciales es una de las herramientas más utilizadas (Jain et al., 2011). La autenticación de personas a partir de imágenes consiste en, extraer rasgos característicos faciales de una persona, estos son almacenados en un vector, el cual servirá para entrenar un clasificador y así poder realizar, posteriormente, la autenticación de la persona. Hay ejemplos muy simples donde se autentican personas utilizando características faciales, como el sistema de seguridad de algunos teléfonos inteligentes, en los que tienen que almacenar previamente el rostro de la persona, para que posteriormente, se pueda realizar una comparación de características entre la imagen almacenada y la imagen que se está capturando en el momento de intentar desbloquear el teléfono (Adjabi et al., 2020).

Otro ejemplo donde se implementa la autenticación de personas utilizando los rasgos faciales es en la zona de abordaje de aeropuertos del primer mundo (Huang \& Zhu, 2021). En estos lugares se utilizan sistemas que contienen algoritmos capaces de poder autenticar personas; utilizando como imagen comparativa, la imagen obtenida de su pasaporte. Como estos ejemplos, hay muchas otras aplicaciones, pero aún se sigue investigando cómo mejorar estos sistemas capaces de autenticar personas a través de sus características faciales. 
Se ha hablado de ejemplos donde autentican personas, pero no se ha mencionado que este proceso consta de diferentes etapas como: detección de rostros, extracción de características, clasificación y finalmente, la etapa de la autenticación de personas (De-la-Torre et al., 2015). El propósito del presente artículo es demostrar que la combinación de características que se propone en conjunto con el clasificador es una alternativa para llevar a cabo el proceso de autenticación de personas. Por tal motivo, en primer lugar, se analizarán diferentes trabajos relacionados a este proceso, acto seguido, se presentará la metodología propuesta y se explicarán a grandes rasgos las diferentes etapas que componen esta metodología. Posteriormente, se expondrán los resultados obtenidos de los diferentes experimentos realizados. Por último, se mostrarán las conclusiones y trabajo futuro.

\section{Trabajos relacionados}

Dentro de la literatura, existen trabajos que emplean diferentes técnicas para la detección de rostros y la extracción de características, esto con el objetivo de tener buenos resultados en el proceso de autenticación. Los clasificadores más utilizados en el estado del arte son: Máquinas de vectores de soporte (Support Vector Machines, SVM), Redes neuronales (Neural Networks, NN), K-Vecinos más cercanos (K-Nearest Neighbors, $K-N N$ ), y AdaBoost (Adaptive Boost). Cabe mencionar que, dentro del estado del arte del procesamiento digital en imágenes faciales, existen dos grandes variantes, las cuales son: el reconocimiento facial, y el reconocimiento de expresiones faciales. A continuación, se describen algunos trabajos al respecto.

Recientemente se han realizado diversas publicaciones centradas en la extracción de rasgos faciales para el reconocimiento de expresiones faciales (Balamurugan \& Suganya, 2021; Dino \& Abdulrazzaq, 2019; Kar et al., 2019; Liu et al., 2020; Reddy et al., 2019; Shah et al., 2020). Por ejemplo, en el trabajo de Balamurugan \& Suganya (2021), para la etapa de extracción de características utilizan la técnica de Histograma Orientado de Gradientes (HOG), mientras que, para la etapa de clasificador, utilizan SVM. Estas técnicas, son empleadas para la detección de las diferentes expresiones faciales en tiempo real, tales como, felicidad, tristeza, enojo, sorpresa, temor, y disgusto.

Otros trabajos como el realizado por Dino \& Abdulrazzaq (2019), emplea el detector de rostros ViolaJones en la primera etapa, seguido de la etapa de extracción de característicos usando HOG. Finalmente exploran tres tipos de clasificadores: SVM, K-NN, y Multilayer Perceptron (MLP), determinando que el mejor resultado obtenido es por la SVM.

Así como en los trabajos ya expuestos, existen múltiples autores que han tomado como base a las SVM como clasificadores, variando su proceso la forma en la cual se extraen las características de las imágenes (Kar et al., 2019; Liu et al., 2020; Reddy et al., 2019; Shah et al., 2020). Por ejemplo, algunos extraen bordes y texturas, otros Local Discriminant Analysis (LDA) o incluso, analizan características geométricas. Otra característica, que tienen en su mayoría estos trabajos, es que utilizan Principal Component Analysis (PCA), para reducir la dimensión de las características al igual que, el costo computacional de las ejecuciones.

Por otro lado, con reconocimiento facial también se identifica una lista importante de trabajos con diferentes enfoques (Almabdy \& Elrefaei, 2019; Bindu \& Manjunathachary, 2019; Chen \& Haoyu, 2019; George et al., 2019; Ghazal \& Abdullah, 2020; Hu \& Cui, 2019; Singh et al., 2020; Vengatesan et al., 2019; Zhang, 2019). Entre los más representativos están aquellos basados en PCA. Por ejemplo, Hu \& Cui (2019), utilizan un operador de máscara diferencial fraccional para describir y analizar las imágenes, luego se extraen las características con PCA, para reconocer las imágenes con una SVM.

Otra aproximación explorada por los autores es la realizada por Bindu \& Manjunathachary (2019), donde se aplica Scale-Invariant Feature Transform (SIFT). Los autores proponen aplicar diferentes kernels para la extracción de características, las cuales son utilizadas para entrenar una SVM y así realizar el proceso de reconocimiento facial.

Una técnica utilizada en el trabajo de Ghazal \& Abdullah (2020) es la de Transformada Rápida de Curvas Discretas, la cual representa una transformación geométrica de múltiples escalas. Ésta es utilizada para extraer características, que, en conjunto con una SVM, son empleadas para el proceso de reconocimiento facial en momentos invariantes.

Al igual que en el reconocimiento de expresiones faciales, HOG se utiliza para reconocimiento facial (George et al., 2019; Singh et al., 2020). En el trabajo de George et al. (2019), los autores hacen una combinación de diferentes canales que sirven como características. Para ello, fueron generados 10 canales: 
6 canales de HOG, 3 canales de LUV (espacio de color), y 1 canal de la magnitud del gradiente. Estos canales/características, son reducidos en dimensión a través de PCA, para después, pasar a la clasificación de una SVM. Por otro lado, el trabajo de Singh et al. (2020), emplea una combinación de Patrones Binarios Locales (Local Binary Patterns, LBP) con HOG para la etapa de extracción de características, ya que han sido dos de las técnicas más utilizadas en aplicaciones en tiempo real. Después de haber extraído las características, se entrena una SVM y se obtiene como resultado el proceso de reconocimiento facial.

Vengatesan et al. (2019) y Zhang (2019), utilizan descriptores de textura, como la Matriz de coocurrencia de niveles de grises (Gray Level Co-Ocurrence Matrix, GLCM) o LBP. Los primeros autores utilizan GLCM, para hacer un reconocimiento facial entre gemelos idénticos, y un filtro de Gabor para resaltar ciertas características en cada una de las imágenes de entrada y así, poder entrenar el clasificador para realizar el proceso del reconocimiento entre los gemelos. Mientras que, Zhang, implementa LBP como características a extraer, que se utilizarán para entrenar una SVM.

Por último, tanto para la etapa de extracción de características como para la etapa de la clasificación se usan aproximaciones basadas en un enfoque conexionista (Redes Neuronales). Almabdy \& Elrefaei (2019) y Chen \& Haoyu (2019), emplean algunas variantes de las NN. Almabdy \& Elrefaei (2019) aplican una Red Neuronal Convolucional (CNN, por sus siglas en inglés) para extraer las características. Al respecto, existen diferentes arquitecturas que se han propuesto y entrenado por diversos autores y que han demostrado tener buenos resultados, como lo son las arquitecturas AlexNet y RasNet-50, las cuales obtienen una serie de características que son usadas para entrenar una SVM. Otro ejemplo de este enfoque es el propuesto por Chen \& Haoyu (2019), que emplean la arquitectura VGG-16. Este modelo es usado para extraer las características de los rostros, después, estas características son reducidas en dimensionalidad mediante la técnica de PCA, para posteriormente, pasar a la etapa de clasificación con una SVM.

\section{Metodología propuesta}

Comúnmente, el proceso de autenticación de personas suele basarse en las etapas: detección de rostros, extracción de características, clasificación, y autenticación de personas (ver Figura 1). Cabe recordar que, en este trabajo, solo se abordan las dos últimas etapas (clasificación y autenticación de personas), refiriendo que las etapas previas ya fueron analizadas y realizadas con anterioridad.

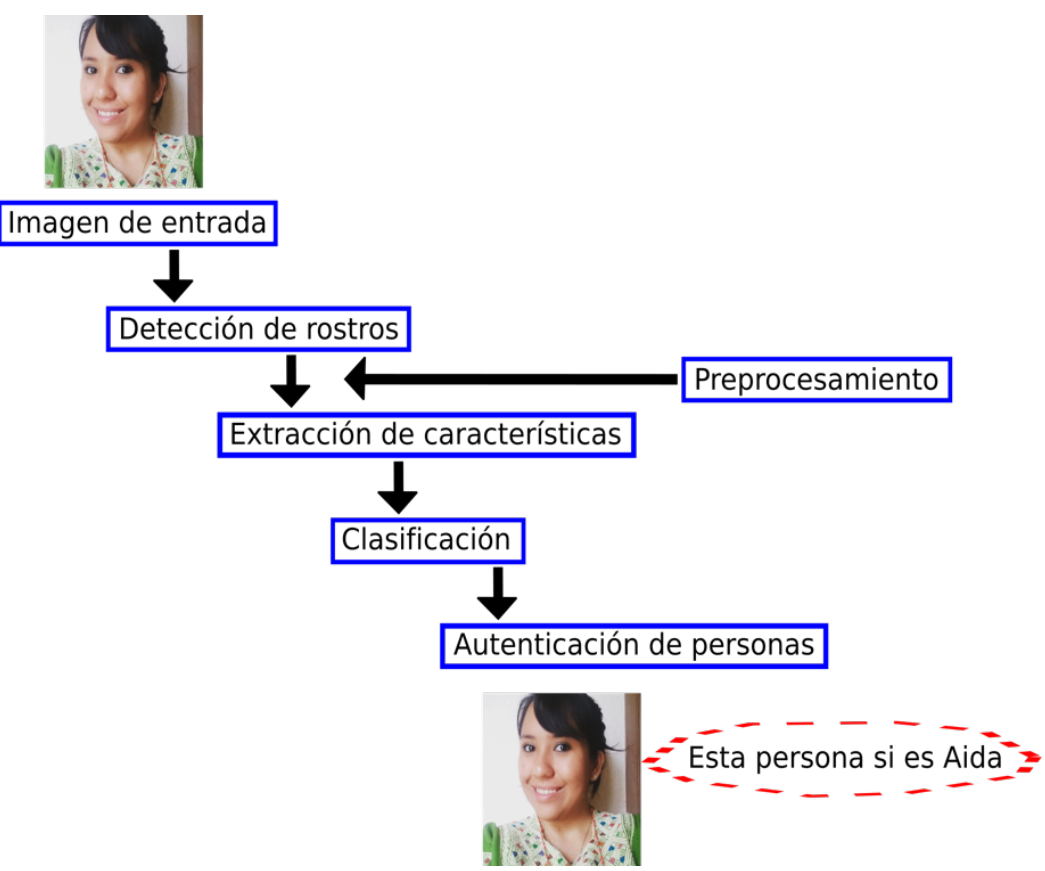

Figura 1. Diagrama del proceso de autenticación de personas. 
La primera etapa del proceso general es la detección de rostros. Para ello se tiene una imagen de entrada, en la cual se detectará el rostro de una persona aplicando una CNN de arquitectura VGG-16 pre-entrenada, la cual puede detectar objetos diversos. Siguiendo con el diagrama, en la etapa de preprocesamiento, se hacen correcciones a las imágenes tanto en rotación como traslación a partir de una matriz, con el fin de tenerlas todas en una misma posición y a un mismo tamaño, de igual manera, se aplican diferentes filtros para corregir la iluminación y el contraste, para después seguir con la etapa de extracción de las características faciales.

Existen diferentes categorías de características que se pueden extraer de la zona del rostro. En este trabajo se extraerán tres categorías, las cuales son: las características basadas en el color, las características basadas en la textura, y las características basadas en la simetría. Se proponen estas categorías, ya que analizan distintas regiones del rostro y aportan diferente información y se complementan entre sí, además de que, analizan dos dominios: el dominio espacial y el dominio frecuencial.

Para obtener un vector característico, se aplican diferentes técnicas de extracción (las cuales se explican en el siguiente apartado). Este vector será utilizado para el entrenamiento de un clasificador SVM. El modelo de entrenamiento obtenido se utilizará en la etapa de autenticación de personas. La aportación de este trabajo es en la etapa de preprocesamiento y la etapa de extracción de características faciales, además de que, la metodología que se propone analiza los rostros de las personas tanto cuando están de frente como de perfil, ya que en la mayoría de los trabajos relacionados se enfocan en las imágenes de frente. En el siguiente apartado, se enlistarán las características extraídas y se presentarán tanto el clasificador utilizado como los resultados obtenidos.

\section{Resultados preliminares}

En este trabajo, se generó una nueva base de imágenes, la cual está conformada de 10 personas (5 mujeres y 5 hombres). Esta base cuenta con alrededor de 30 imágenes por persona. Estas imágenes fueron tomadas por las mismas personas en diferentes escenarios y a diferentes distancias. El tamaño de las imágenes capturadas es de $256 \times 256$. Cabe mencionar, que las imágenes de la base de datos que se van a analizar han recibido un preprocesamiento, el cual consiste en una corrección de pose y escalamiento. Este preprocesamiento, se realiza con el fin de tener todas las imágenes en una misma posición (sobre el eje z) y a una misma escala, para posteriormente, pasar a una etapa previa de la extracción de características, la etapa de segmentación. La segmentación, se hace con el objetivo de siempre tener las mismas regiones del rostro. En la Figura 2, se muestra un diagrama general de este preprocesamiento.

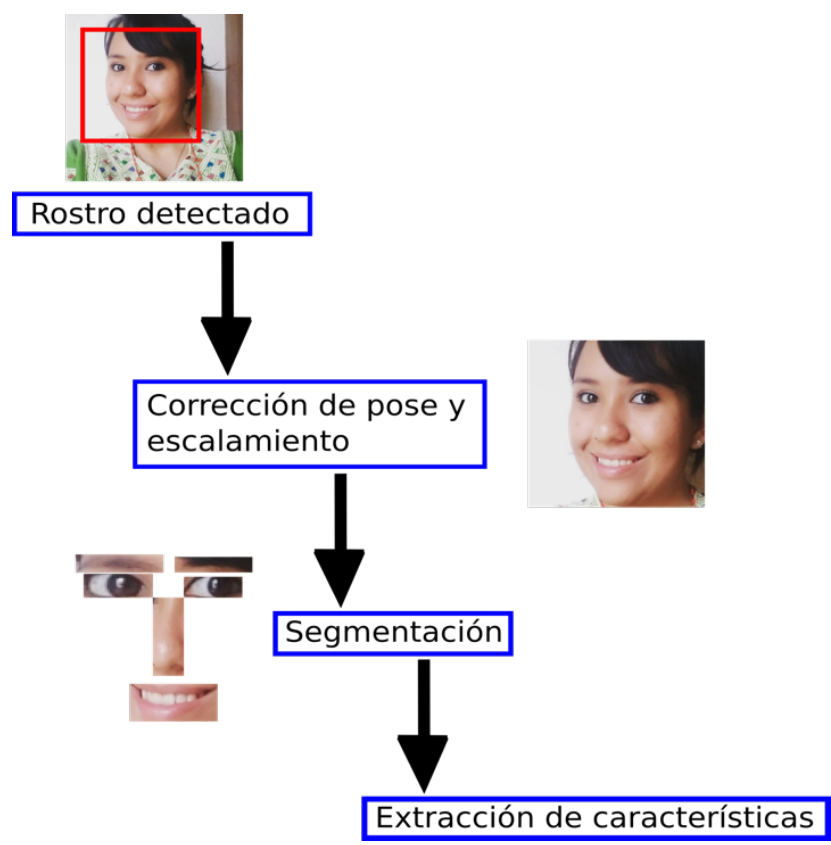

Figura 2. Diagrama general de la etapa de preprocesamiento. 
A continuación, se hace una breve explicación sobre estas dos etapas previas a la extracción de características. Para la etapa de corrección de pose y escalamiento, se utiliza una técnica para identificar Landmarks, el cual utiliza una plantilla para detectar 5 o 68 puntos de referencia dentro del rostro (Sagonas et al., 2013). Para nuestro trabajo de investigación, se utiliza la plantilla con los 68 puntos de referencia. Estos puntos corresponden a diferentes zonas de interés (ROIs, por sus siglas en inglés), las cuales son: las cejas, los ojos, la nariz, y la boca.

Una vez localizadas estas zonas de interés, se genera una matriz de transformación, la cual consta de una parte para rotar el rostro, otra parte para trasladar el rostro dentro de la imagen y, por último, una parte para escalar el rostro. Esta matriz se genera a partir de localizar el centro de los ojos, después, se determina la posición a la que se desea colocar el rostro, para así poder rotar y/o trasladar la cara dentro de la imagen. En la Figura 3, se muestran algunos de los resultados de este proceso de corrección de pose y escalamiento.

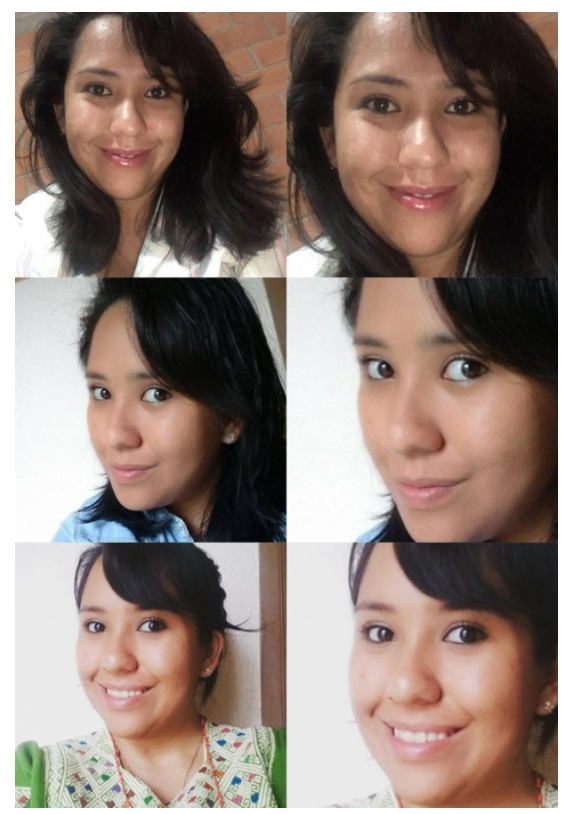

Figura 3. Ejemplos de la corrección de pose y escalamiento. Imagen original (columna izquierda), rostro corregido (columna derecha).

Después de haber realizado el proceso de corrección de pose y escalamiento, se continúa con el proceso de segmentación. Se utiliza la misma plantilla de los Landmarks, para localizar los 68 puntos de referencia. La imagen del rostro se va segmentando en cada uno de esos ROIs. En la Figura 4, se pueden observar algunos de los resultados obtenidos para la parte de la segmentación del rostro. 


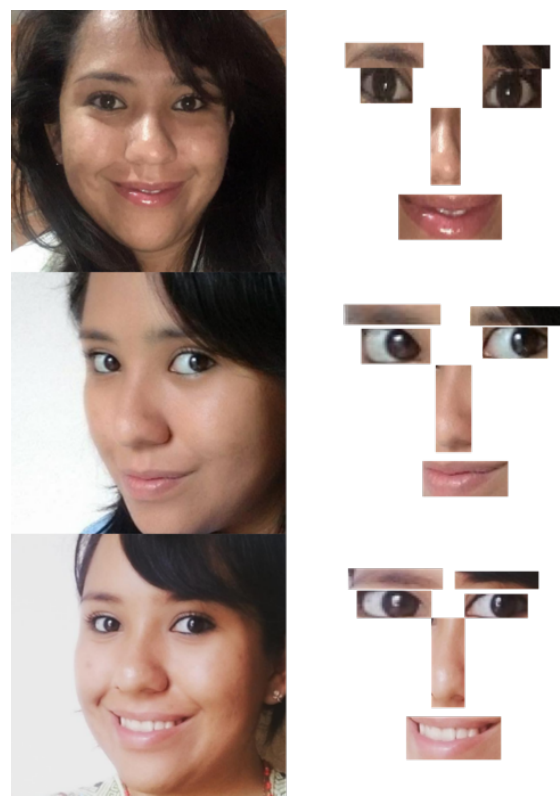

Figura 4. Ejemplos de la etapa de segmentación. Rostro corregido (columna izquierda), rostro segmentado (columna derecha).

Ya segmentado el rostro, a cada una de las zonas obtenidas se aplica una ecualización del histograma, con el fin de obtener las imágenes con un brillo y contraste uniforme. Las características extraídas para cada una de las categorías se enlistan en la Tabla 1 .

Tabla 1. Características extraídas de cada una de las diferentes áreas de análisis (basadas en color, basadas en textura y basadas en simetría).

\begin{tabular}{|l|l|}
\hline \multicolumn{2}{|c|}{ Área de análisis } \\
Características basadas en color & $\begin{array}{l}\text { Desviación estándar (R,G,B), Varianza (R,G,B), Entropía (R,G,B), } \\
\text { Desviación estándar (H,S,V), Varianza (H,S,V), y Entropía (H,S,V). }\end{array}$ \\
\hline Características basadas en textura & Matriz de Coocurrencia, Transformada de Fourier y Filtro de Gabor. \\
\hline Características basadas en simetría & Ancho del rostro, ancho de los ROIs y distancia entre los ROIs. \\
\hline
\end{tabular}

Para extraer las características, se realiza un proceso diferente para cada categoría. Para las características basadas en color, las imágenes se dividen en tres canales $(\mathrm{R}, \mathrm{G}, \mathrm{B})$ y posteriormente, se hace una conversión de color a HSV. En cada uno de estos canales (R,G,B,H,S,V), se extraen diferentes métricas estadísticas como: desviación estándar, varianza, y entropía.

Siguiendo con la misma dinámica, para las características basadas en textura, la imagen original es convertida a una imagen en escala de grises. De esta imagen resultante, se extrae la matriz de co-ocurrencia en niveles de grises, se obtiene la Transformada de Fourier y, por último, se aplica el filtro de Gabor. Para cada una de estas técnicas, se logran diferentes características. Por ejemplo, de la matriz de co-ocurrencia, se obtiene sus diferentes propiedades como: contraste, energía, correlación, entropía, entre otras.

Para el caso de la Transformada de Fourier, se analiza la magnitud del espectro y de este, se puede obtener la media y varianza. Por último, para el filtro de Gabor, de la imagen resultante se saca la media y varianza. Mientras que, para las características basadas en simetría, de las imágenes segmentadas se mide la longitud de las ROIs, es decir, el ancho de las cejas, el ancho de los ojos, el ancho de la nariz, y la boca.

Como se menciona en los trabajos relacionados, uno de los clasificadores más utilizados dentro del aprendizaje automático es SVM. Este clasificador trabaja con diferentes kernels, como lo son: rbf, linear, poly, sigmoid, y precomputed. La función del kernel es proyectar la información de entrada a un espacio de mayor dimensión, para aumentar la capacidad computacional de las máquinas, esto debido a que no siempre los datos van a tener solo dos variables predictivas o no van a ser completamente separables. Para uso de los experimentos realizados en este trabajo, se utiliza el kernel linear.

Los experimentos que se realizaron se comprenden de la siguiente manera. Se utilizaron las 30 imágenes de las 10 personas que conforman la base de datos que se capturó. Después de haber realizado la etapa de 
corrección de pose y escalamiento, se hace la segmentación de los rostros ya corregidos. A cada una de las zonas segmentadas, se extraen las características anteriormente mencionadas. Estas se almacenan en un vector, el cual será utilizado para entrenar el clasificador. Del vector, se dividen los datos en un $75 \%$ para la parte de entrenamiento y el restante $25 \%$ para la parte de pruebas. Se hicieron experimentos con diferente número de personas (de 2 personas a 10 personas) y con diferente número de características.

Para determinar el conjunto de características, se hicieron experimentos previos, los cuales constaban de ir combinando diferentes características de las distintas categorías que se mencionan al principio. Al final de estos experimentos, se determinaron cuáles serían las combinaciones de características, que en conjunto aportarían más información en la etapa de entrenamiento del clasificador. Puede haber combinaciones en las que, solamente se tomen en cuenta las características basadas en color y las basadas en simetría, o, por otro lado, no se tomen en cuenta todas las características basadas en color, pero si todas las características basadas en textura, y las características basadas en simetría. En la Tabla 2, se enlistan la menor y la mayor cantidad de características combinadas.

Tabla 2. Combinaciones de las diferentes características extraídas de cada una de las áreas de análisis.

\begin{tabular}{|l|l|}
\hline 15 características & $\begin{array}{l}\text { Características } \\
\text { (H,S,V), Matriz de Coocurrencia (Disimilitud, Entropía), Transformada de } \\
\text { Fourier (Media de la magnitud del espectro, Varianza de la magnitud del } \\
\text { espectro), distancia de los ROIs, y distancia entre los ROIs. }\end{array}$ \\
\hline 36 características & $\begin{array}{l}\text { Desviación estándar (R,G,B), Varianza (R,G,B), Entropía (R,G,B), Valor } \\
\text { mínimo (H,S,V), Valor máximo (H,S,V), Desviación estándar (H,S,V), }\end{array}$ \\
& $\begin{array}{l}\text { Varianza (H,S,V) y Entropía (H,S,V), Matriz de Co-ocurrencia (Contraste, } \\
\text { Energía, Correlación, Homogeneidad, Disimilitud, Entropía), Transformada } \\
\text { de Fourier (Media de la magnitud del espectro, Varianza de la magnitud del } \\
\text { espectro), Filtro de Gabor (Media del filtro, Varianza del filtro), distancia de } \\
\text { los ROIs, y distancia entre los ROIs. }\end{array}$ \\
\hline
\end{tabular}

Como resultado de la clasificación, se obtiene el porcentaje de precisión de cada persona que conforma el experimento, la matriz de confusión. y la precisión global. En la Tabla 3, se muestran los resultados obtenidos para los diferentes experimentos realizados. En este caso, solamente se presentan los porcentajes de la precisión global.

Tabla 3. Resultados de la etapa de clasificación, utilizando una SVM y el kernel linear

\begin{tabular}{|l|c|c|}
\multicolumn{1}{c|}{\begin{tabular}{c|c|} 
Experimento \\
2 personas
\end{tabular}} & $\begin{array}{c}\text { Precisión global con 15 } \\
\text { características }\end{array}$ & $98 \%$ \\
\hline 4 personas & $92 \%$ & $94 \%$ \\
\hline 6 personas & $84 \%$ & $73 \%$ \\
\hline 8 personas & $78 \%$ & $71 \%$ \\
\hline 10 personas & $73 \%$ & $73 \%$ \\
\hline
\end{tabular}

Para realizar una comparación de la precisión de las características propuestas en este trabajo con técnicas utilizadas dentro del estado del arte, se hicieron experimentos con otras técnicas de extracción de rasgos faciales: PCA y LBP. En la Tabla 4, se muestran los resultados obtenidos de experimentos donde los rostros de las personas están de frente a la cámara. Para esta experimentación, se utilizó el mismo conjunto de imágenes que se generó previamente, el mismo clasificador SVM, y mismo equipo de cómputo. Cabe mencionar, que, en la columna de características propuestas, se muestran los mejores resultados de la Tabla 3 . 
Tabla 4. Tabla comparativa de técnicas de extracción de características en el estado del arte para rostros de frente.

\begin{tabular}{|l|c|c|c|}
\multicolumn{1}{r}{ Experimento } & $\begin{array}{c}\text { Precisión global } \\
\text { caracteristicas } \\
\text { propuestas }\end{array}$ & Precisión global PCA & Precisión global LBP \\
\hline 2 personas & $98 \%$ & $100 \%$ & $86 \%$ \\
\hline 4 personas & $94 \%$ & $100 \%$ & $80 \%$ \\
\hline 6 personas & $78 \%$ & $96 \%$ & $60 \%$ \\
\hline 8 personas & $73 \%$ & $93 \%$ & $61 \%$ \\
\hline 10 personas & $73 \%$ & $88 \%$ & $61 \%$ \\
\hline
\end{tabular}

Mientras que, en la Tabla 5 se muestran los resultados obtenidos de los experimentos donde los rostros de las personas están de perfil con respecto a la cámara. Al igual que en la experimentación previa, se utilizó el mismo conjunto de imágenes que se generó, el mismo clasificador SVM, y mismo equipo de cómputo.

Tabla 5. Tabla comparativa de técnicas de extracción de características en el estado del arte para rostros de perfil.

\begin{tabular}{|c|c|c|c|}
\hline Experimento & $\begin{array}{c}\text { Precisión global } \\
\text { características } \\
\text { propuestas }\end{array}$ & Precisión global PCA & Precisión global LBP \\
\hline 2 personas & $66 \%$ & $60 \%$ & $57 \%$ \\
\hline 4 personas & $80 \%$ & $47 \%$ & $41 \%$ \\
\hline 6 personas & $61 \%$ & $56 \%$ & $39 \%$ \\
\hline 8 personas & $50 \%$ & $48 \%$ & $25 \%$ \\
\hline 10 personas & $76 \%$ & $48 \%$ & $27 \%$ \\
\hline
\end{tabular}

\section{Conclusiones y trabajo futuro}

Como se puede ver en los resultados obtenidos, conforme se aumentó el número de personas, el porcentaje de precisión global fue variando. Esto es debido a las características de las imágenes, porque como se menciona, esta base de imágenes fue generada por 10 personas, las cuales tomaron sus propias fotos, por tal motivo, ninguna foto fue tomada bajo las mismas condiciones o con el mismo dispositivo (teléfono celular). Esto es un factor que se va a tomar en consideración para un trabajo a futuro.

Haciendo un análisis de las Tablas 4 y 5, cuando se extraen las características en las imágenes de perfil, los porcentajes de precisión son más altos con el conjunto de características que se propone a comparación de las otras dos técnicas. Mientras que, para los casos de imágenes de frente, la técnica que obtiene mejores porcentajes de precisión es PCA, seguido por la combinación de características que se propone. Cabe mencionar que, una diferencia entre el conjunto de características que se propone en este trabajo y las dos técnicas con las que se compara, es que éstas analizan toda la imagen del rostro, mientras que, en la propuesta se analizan las características en el rostro segmentado, es decir, en las diferentes ROIs (ojos, cejas, nariz, boca). En este sentido, habrá zonas del rostro que aporten información y no sean tomadas en cuenta dentro de las ROIs.

Como conclusión, el conjunto de características propuesto trabaja bien con ambos casos, tanto con imágenes de frente como con imágenes de perfil, pero se tiene que seguir trabajando para mejorar los resultados obtenidos. Por tal motivo, se seguirán analizando las diferentes características que se proponen y poder determinar, cuáles son las que aportan más información para la etapa de clasificación, con el objetivo de aumentar la precisión en la clasificación. De igual manera, se propone realizar experimentos con bases de datos públicas y poder medir la eficiencia del conjunto de características que se propone en este trabajo.

\section{Declaración de conflicto de intereses}

Los autores declaran no tener conflicto de intereses con respecto a la investigación, autoría o publicación de este artículo. 


\section{Financiación}

El primer autor, cuyo número de becario es 331482, agradece al CONACYT (Consejo Nacional de Ciencia y Tecnología) el apoyo brindado de la beca de estudios de posgrado, derivando la publicación del presente artículo.

\section{ORCID iD}

Aida A. Aparicio-Arroyo iD https://orcid.org/0000-0002-3547-2433
Ivan Olmos-Pineda iD https://orcid.org/0000-0003-1698-000X
J. Arturo Olvera-López (iD https://orcid.org/0000-0003-0639-1463

\section{Referencias}

Adjabi, I., Ouahabi, A., Benzaoui, A., \& Taleb-Ahmed, A. (2020). Past, Present, and Future of Face Recognition: A Review. Electronics, 9(8). https://doi.org/10.3390/electronics9081188

Almabdy, S., \& Elrefaei, L. (2019). Deep Convolutional Neural Network-Based Approaches for Face Recognition. Applied Sciences, 9(20). https://doi.org/10.3390/app9204397

Balamurugan, A., \& Suganya, B. (2021). An Efficient Real Time Face Expression Identification System Using SVM. Journal of Physics: Conference Series, 1916(1). https://doi.org/10.1088/1742-6596/1916/1/012229

Bindu, H., \& Manjunathachary, K. (2019). Kernel-based scale-invariant feature transform and spherical SVM classifier for face recognition. Journal of Engineering Research, 7(3), 142-160. https://kuwaitjournals.org/jer/index.php/JER/article/view/4177

Chen, H., \& Haoyu, C. (2019). Face Recognition Algorithm Based on VGG Network Model and SVM. Journal of Physics: Conference Series, 1229. https://doi.org/10.1088/1742-6596/1229/1/012015

De-la-Torre, M., Granger, E., Radtke, P. V. W., Sabourin, R., \& Gorodnichy, D. O. (2015). Partially-supervised learning from facial trajectories for face recognition in video surveillance. Information Fusion, 24. https://doi.org/10.1016/j.inffus.2014.05.006

Dino, H. I., \& Abdulrazzaq, M. B. (2019, April). Facial Expression Classification Based on SVM, KNN and MLP Classifiers. 2019 International Conference on Advanced Science and Engineering (ICOASE). https://doi.org/10.1109/ICOASE.2019.8723728

George, M., Sivan, A., Jose, B. R., \& Mathew, J. (2019). Real-time single-view face detection and face recognition based on aggregate channel feature. International Journal of Biometrics, 11(3). https://doi.org/10.1504/IJBM.2019.100829

Ghazal, M. T., \& Abdullah, K. (2020). Face recognition based on curvelets, invariant moments features and SVM. TELKOMNIKA (Telecommunication Computing Electronics and Control), 18(2), 733-739. https://doi.org/10.12928/telkomnika.v18i2.14106

Hu, L., \& Cui, J. (2019). Digital image recognition based on Fractional-order-PCA-SVM coupling algorithm. Measurement, 145. https://doi.org/10.1016/j.measurement.2019.02.006

Huang, H., \& Zhu, J. (2021). A Short Review of the Application of Machine Learning Methods in Smart Airports. Journal of Physics: Conference Series, 1769. https://doi.org/10.1088/1742-6596/1769/1/012010

Jain, A. K., Ross, A. A., \& Nandakumar, K. (2011). Introduction to Biometrics. Springer US. https://doi.org/10.1007/978-0-387-77326-1

Kar, N. B., Babu, K. S., Sangaiah, A. K., \& Bakshi, S. (2019). Face expression recognition system based on ripplet transform type II and least square SVM. Multimedia Tools and Applications, 78(4). https://doi.org/10.1007/s11042-017-5485-0

Liu, X., Cheng, X., \& Lee, K. (2020). GA-SVM based Facial Emotion Recognition using Facial Geometric Features. IEEE Sensors Journal, 21(10), 11532-11542. https://doi.org/10.1109/JSEN.2020.3028075

Reddy, C. V. R., Reddy, U. S., \& Kishore, K. V. K. (2019). Facial Emotion Recognition Using NLPCA and SVM. Traitement Du Signal,36(1). https://doi.org/10.18280/ts.360102

Sagonas, C., Tzimiropoulos, G., Zafeiriou, S., \& Pantic, M. (2013). A Semi-automatic Methodology for Facial Landmark Annotation. Proceedings of the IEEE Conference on Computer Vision and Pattern Recognition (CVPR) Workshops.

Shah, J. H., Sharif, M., Yasmin, M., \& Fernandes, S. L. (2020). Facial expressions classification and false label reduction using LDA and threefold SVM. Pattern Recognition Letters, 139. https://doi.org/10.1016/j.patrec.2017.06.021 
Singh, S., Singh, D., \& Yadav, V. (2020). Face Recognition Using HOG Feature Extraction and SVM Classifier. International Journal of Emerging Trends in Engineering Research, 8(9). https://doi.org/10.30534/ijeter/2020/244892020

Vengatesan, K., Kumar, A., Karuppuchamy, V., Shaktivel, R., \& Singhal, A. (2019, December). Face Recognition of Identical Twins Based On Support Vector Machine Classifier. 2019 Third International Conference on I-SMAC (IoT in Social, Mobile, Analytics and Cloud) (I-SMAC). https://doi.org/10.1109/I-SMAC47947.2019.9032548

Zhang, B. (2019). Distributed SVM face recognition based on Hadoop. Cluster Computing, 22(S1). https://doi.org/10.1007/s10586-017-1330-5 